\title{
Bilateral Simultaneous Testicular Torsion in a Newborn: Report of a Case
}

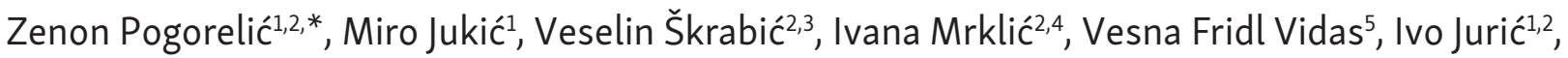 \\ Dubravko Furlan ${ }^{1,2}$
}

\begin{abstract}
Introduction: Testicular torsion is a urological emergency. If not recognized in time, this condition may result in ischaemic injury and loss of testis. Simultaneous bilateral neonatal testicular torsion is extremely rare and is usually misdiagnosed.

Case report: We report a case of a male newborn, who presented with bilateral scrotal swelling and redness of the scrotum. Doppler ultrasound supported the diagnosis of bilateral testicular torsion, with an absent blood flow signal on the right side and a weak signal on the left side. Testicular exploration through scrotal incision was performed and bilateral testicular torsion was found. Right testis was grossly gangrenous, and right orchiectomy was performed. Left testicle was dark but showed recovery after detorsion with some bleeding from incised tunica albugenia. Fixation of the left testicle was performed. At six month follow-up, the left testis showed signs of atrophy and hormonal assay showed very low testosterone and elevated $\mathrm{LH}$ and $\mathrm{FSH}$, suggesting hypogonadism.

Conclusions: Management of neonatal testicular torsion is a matter of controversy. Testicular torsion results into acute ischemia and urgent surgical exploration is the key point of management. Although the possibility of salvaging the involved testicles is usually very low it is hard to justify a passive approach to a bilateral torsion resulting in such a devastating condition as anorchia.
\end{abstract}

\section{KEYWORDS}

testicular torsion; neonatal torsion; newborn; bilateral testicular torsion; simultaneous testicular torsion

\author{
AUTHOR AFFILIATIONS \\ ${ }^{1}$ Department of Pediatric Surgery, University Hospital of Split, Split, Croatia \\ ${ }^{2}$ University of Split, School of Medicine, Split, Croatia \\ ${ }^{3}$ Department of Pediatrics, University Hospital of Split, Split, Croatia \\ ${ }^{4}$ Department of Pathology, University Hospital of Split, Split, Croatia \\ ${ }^{5}$ Department of Radiology, University Hospital of Split, Split, Croatia \\ * Department of Pediatric Surgery, University Hospital of Split, Spinčićeva 1, 21000 Split, Croatia; e-mail: zpogorelic@gmail.com
}

Received: 17 April 2017

Accepted: 11 June 2017

Published online: 5 February 2018

Acta Medica (Hradec Králové) 2017; 60(3): 120-123

https://doi.org/10.14712/18059694.2018.4

(c) 2017 The Authors. This is an open-access article distributed under the terms of the Creative Commons Attribution License (http://creativecommons.org/licenses/by/4.0), which permits unrestricted use, distribution, and reproduction in any medium, provided the original author and source are credited. 


\section{INTRODUCTION}

Testicular torsion refers to the torsion of the spermatic cord structures and subsequent loss of the blood supply to the ipsilateral testicle. This is a urological emergency; early diagnosis and treatment are esential for saving the testicle and preserving future fertility. Unfortunately there may be a lack of awareness among physicians or parents with regard to this urological emergency, and in most cases the diagnosis is deferred (1). Annual incidence of testicular torsion is 4.5 in 100,000 males 1-25 years of age (2). It can occur at any age but usually occurs in young males, with a bimodal incidence in the pediatric population: during the first year of life, and between the ages of 13 and $16(1,2)$. Bilateral cases account for $2 \%$ of all torsions, and simultaneous bilateral testicular torsion is extremely rare (3). It is a commonly held belief that a testicle torsed for longer than $6 \mathrm{~h}$ is outside the survival time-frame (1-3). If treated within $6 \mathrm{~h}$ of the presenting pain, there is a good chance of saving the affected testicle, as $90-100 \%$ testicles will be saved. If treated within 6-12 h $20-50 \%$ testicles will be saved and if treated within $12-24 \mathrm{~h}$ 0-10\% testicles will be saved (2). Neonatal testicular torsion is a rare event with controversies regarding its etiology, presentation, surgical management and sequelae (4). It may be unilateral or bilateral. Bilateral torsion may be synchronous or asynchronous. The time when the torsion occurred is very difficult to determine; it has been reported to occur antenatally as well postnatally (5). Usually it affects healthy full term newborns, but it has been reported in preterm infants also. Definitive diagnosis is difficult to make without scrotal exploration $(4,5)$. The pediatric urologist or pediatric surgeon is usually consulted in the first few hours of life and is faced with formulating the management plan. There is much controversy regarding the optimal management.

Here, we present a case of simultaneous bilateral testicular torsion of the testicles in a newborn.

\section{CASE REPORT}

A male newborn, weighing $3430 \mathrm{~g}$, was the product of a full-term, uncomplicated vaginal delivery. There were no signs of distress and no abnormalities were noted except diffuse swelling and redness of the scrotum, more evident on right side. The testes were hard and tender on palpation. The abdomen was soft and not tender on palpation. Bowel sounds were presented and there was no evidence of inguinal hernias. The white blood cell count was $18.40 \times 10^{9} / 1$ (normal range; $6.2-17.8 \times 10^{9} / 1$ ) and $C$ reactive protein level was $16.6 \mathrm{mg} / \mathrm{l}$ (normal range; 0.1-4.1 mg/l). All other laboratory examinations showed normal values. An ultrasound of the abdomen showed normal findings. Doppler ultrasound of the scrotum, showed heterogeneous tissue image of the right testicle. Hydrocele with absent blood flow of the right testis was also found (Fig. 1a). Left testicle was swollen and peripheral subcapsular arterial blood flow was presented (Fig. 1b). Bilateral scrotal exploration was performed through scrotal incision and revealed bilateral testicular torsion. A $720^{\circ}$ counterclock-
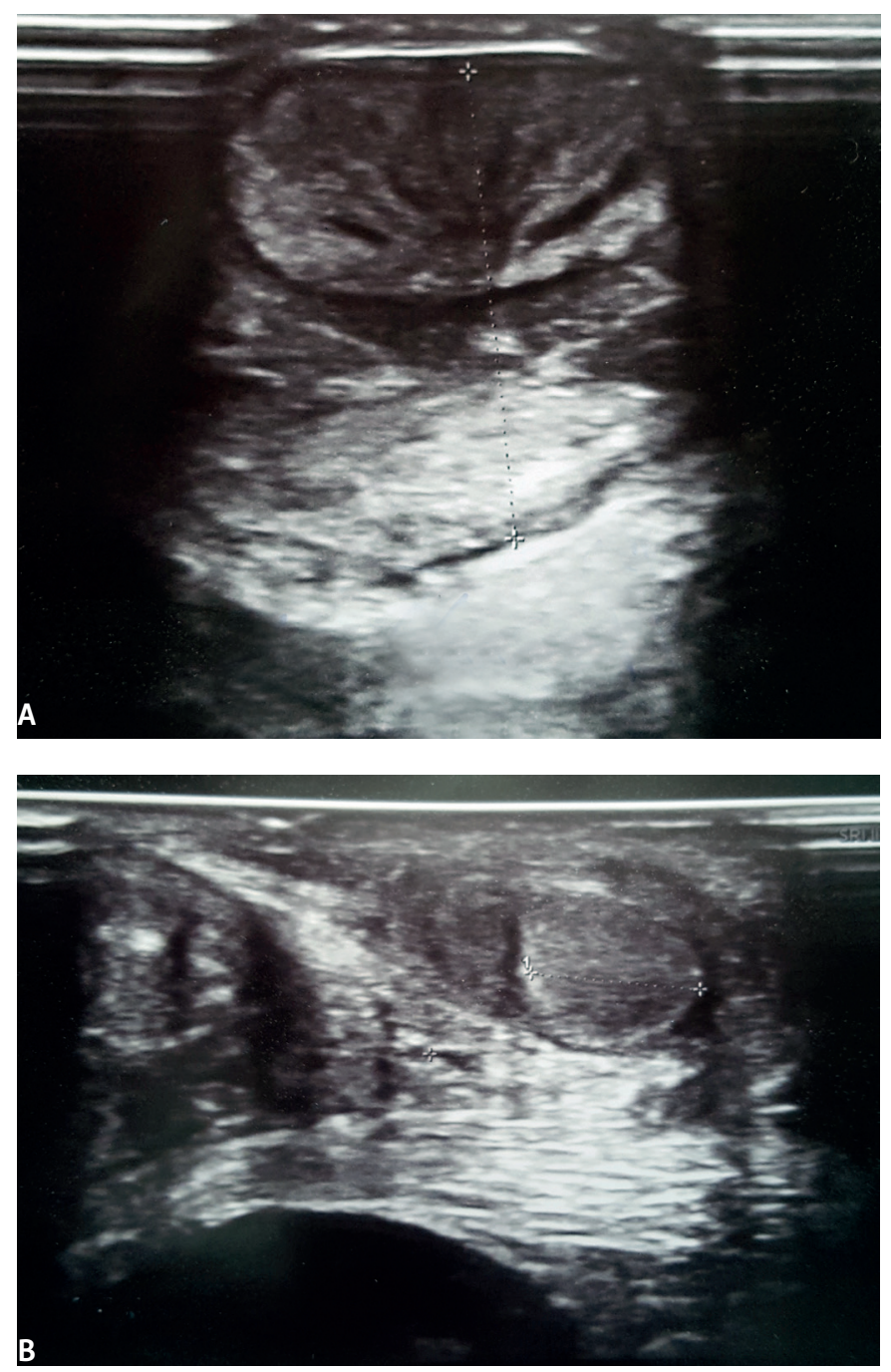

Fig. 1: Ultrasound of the scrotum: A) Heterogeneous hypoehogenic tissue of the right testis; B) Swollen left testis.

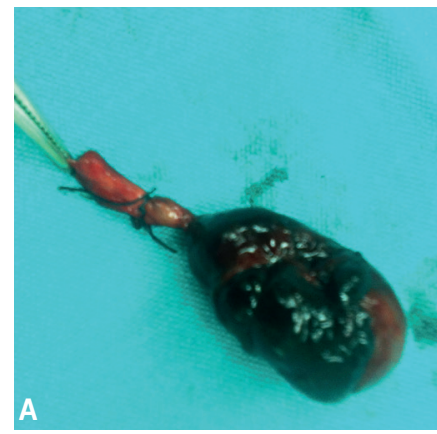

Fig. 2: Intraoperative findings: A) Removed gangrenous right testis; B) Left testis - doubt vitality.

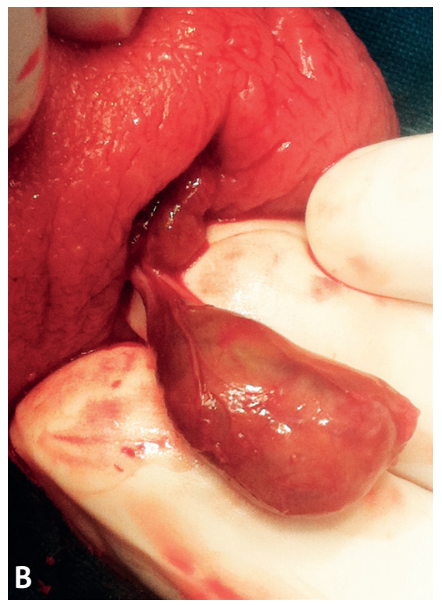

wise extra-vaginal torsion of the right testicle was found with obvious gangrene of the right testicle. Thrombosis of the spermatic blood vessels distally to the site of torsion was also found (Fig. 2a). The left testicle was found to have a $270^{\circ}$ counterclockwise extravaginal torsion, testicle was dark but showed recovery after detorsion with some bleeding from incised tunica albugenia (Fig. 2b). Right orchiectomy was performed with fixation of the left testicle. 


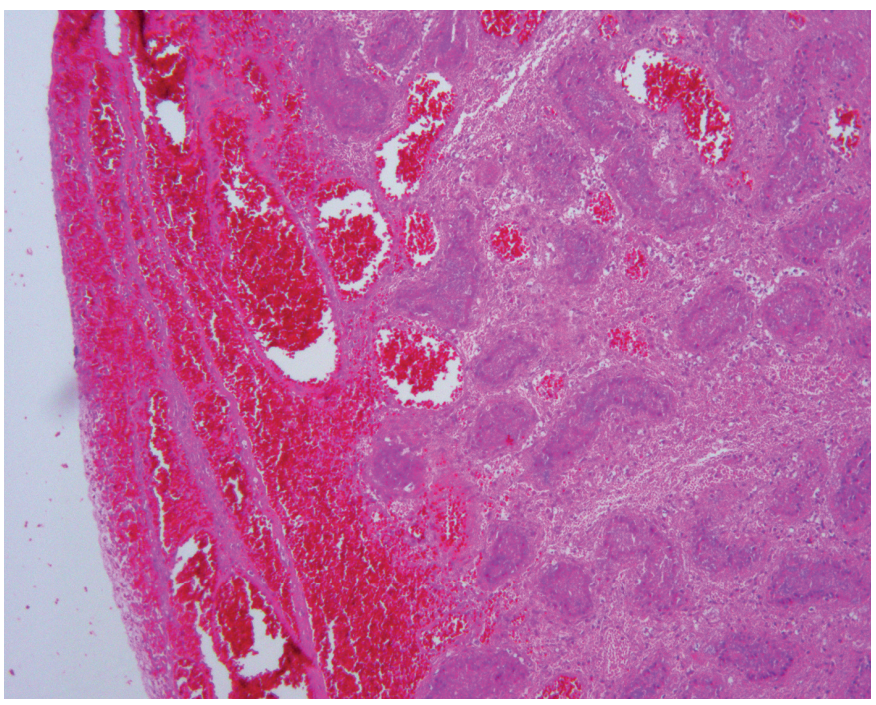

Fig 3: Necrotic testicular tissue, permeated with blood, and severe stagnation of blood in blood vessels (HE x 100).

Histological examination confirmed hemorrhagic necrosis of right testicle (Fig. 3). Follow-up at one month showed that the left testicle was smaller in size without detected testicular blood flow by Doppler ultrasound. However, at six month follow-up, the left testicle showed signs of atrophy and hormonal assay showed very low testosterone and elevated LH and FSH, suggesting primary hypogonadism. The patient was referred to an endocrinologist for further treatment and possible substitution of testosterone.

\section{DISCUSSION}

Both intrauterine and postnatal testicular torsion result with extra-vaginal torsion which is a different entity than the intra-vaginal type but has the same devastating consequences if not diagnosed and managed in time. Testicular torsion results with acute ischemia and its sequelae such as abnormality of testicular function and fertility. Urgent surgical exploration and fixation of the other testis are the key points in the management. Neonatal testicular torsion represents about $12 \%$ of all testicular torsions during infancy (4). It is thought that the majority (70\%) of perinatal torsions are present at delivery and $30 \%$ develop postnatally in the first month of life (6). The clinical features are usually present since birth, but are often unnoticed on time. Epididymitis, scrotal hemathoma or pyocele may simulate testicular torsion in the neonate. Diagnosis is in many cases made based on a high degree of suspicion and from the clinical features alone. Color Doppler ultrasound, testicular isotope scan or MRI, may support the clinical diagnosis, but rarely preclude scrotal exploration especially in the neonate $(6,7)$. Controversy exists concerning the urgency of exploration. Many studies recommend that in newborns who present with suspected unilateral testicular torsion within the first 30 days of age, the risks associated with early surgery and anesthesia outway the prospect of salvaging the testis (4). It is now clear that the potential untoward anesthetic risks involving an otherwise healthy neonate quoted in prior studies are outdated and are not consistent with the current standards of pediatric anesthetic practice (8). There is difference between an acute torsion presenting after birth and the more common entity of the long standing event of antenatal testicular torsion. Das and Singer reported the results of a survey of pediatric urologists and literature review and concluded that early operative intervention with orchiectomy and contralateral orchidopexy was the preferred management strategy for cases of suspected neonatal torsion because surgical exploration was the only definitive way to establish the diagnosis and rule out other potential pathological conditions. They also found that in boys with neonatal torsion, at least $28 \%$ occurred postnatally (6). This suggests a potentially greater rate of testicular salvage than the disappointing results previously reported. Pinto et al. reported salvage rate of $20 \%$ with emergent surgical exploration (9). While there is a theoretical possibility that retention of the infarcted testis may result in some hormonal production, in bilateral testicular torsion it is wise to preserve the least infarcted testicle. Another reason to remove the infarcted testicle is to minimize the chance of aptoposis of the germinal epithelium in the contralateral testicle (5). Many authors adopted the policy of early surgical intervention $(5,8,10,11)$. John et al. reviewed the literature on neonatal testicular torsion. They described 77 boys treated for neonatal torsion, and no testicles were salvaged (12). This dismal outcome underlines that immediate surgical exploration, although commonly performed may not save torted testicles. Reports of neonatal simultaneous bilateral testicular torsion are limited on few case reports, mostly describing lack of diagnosis and poor outcome $(5,10-12)$. Clinical judgement by a pediatric surgeon or urologist is of outmost importance, not the ultrasound. Although the prognosis for these patients is poor, an elective delay in operative intervention seems to be inappropriate from medical and medicolegal point of view. Taking the risk into consideration, bilateral emergent exploration is still strongly advised in all neonates.

\section{CONCLUSIONS}

Neonatal bilateral perinatal testicular torsion is a rare event and it represents a true emergency due to the high risk of hypogonadism. Establishing a correct and fast diagnosis as well as the emergency bilateral surgical exploration are essential in the attempt to save the testicular function. Our recommendation is that in any suspicion of bilateral perinatal torsion of the testicles a bilateral exploration be carried out.

\section{REFERENCES}

1. Pogorelić Z, Mustapić K, Jukić M, et al. Management of acute scrotum in children: a 25 -year single center experience on 558 pediatric patients. Can J Urol 2016; 23: 8594-8601.

2. Pogorelić Z, Mrklić I, Jurić I, et al. Testicular torsion in the inguinal canal in children. J Pediatr Urol 2013; 9:793-797.

3. Saxena A, Castellani C, Ruttenstock E, et al. Testicular torsion: a 15year single-centre clinical and histological analysis. Acta Paediatr 2012; 101: e282-286.

4. Riaz-Ul-Haq M, Mahdi DE, Elhassan EU. Neonatal testicular torsion; a review article. Iran J Pediatr 2012; 22: 281-289. 
5. Ryken TC, Turner JW, Haynes T. Bilateral testicular torsion in a preterm neonate. J Urol 1990; 143: 102-103.

6. Das S, Singer A. Controversies of perinatal torsion of the spermatic cord: a review survey and recommendations. J Urol 1990; 143 231-233.

7. Pepe P, Panella P, Pennisi M, et al. Does color Doppler sonography improve the clinical assessment of patients with acute scrotum. Eur J Radiol 2006; 60: 120-124.

8. Olguner M, Akgur FM, Aktug M, et al. Bilateral asynchronous perinatal testicular torsion: a case report. J Pediatr Surg 2000; 35: 1348-1349.
9. Pinto KJ, Noe HN, Jerkin GR. Management of neonatal testicular torsion. J Urol 1997; 158: 1196-1197.

10. Baglaj M, Carachi R. Neonatal bilateral testicular torsion: a plea for emergency exploration. J Urol 2007; 177: 2296-2299.

11. Al-Salem AH. Intrauterine testicular torsion: a surgical emergency. J Pediatr Surg 2007; 42: 1887-1891.

12. John CM, Kooner G, Mathew ED, et al. Neonatal testicular torsion a lost cause. Acta Paediatrica 2008; 97: 502-504. 